The treatment of electrolysis is carried out mainly by physicians of non-surgical tendencies. That it has done good in the hands of such men as Newman of New York, we can have no doubt. Failure to obtain results should be charged up, not so much to the method as to the operator. This statement applies with equal force to the cutting operation and dilatation without previous urethrotomy. We must remember that others may accomplish what we do not, and when we compare methods, we usually are experienced only in one or the other way; for the balance, we are compelled to be satisfied with reasoning a priori. Keyes" says: "I believe that a strong current is full of danger, both immediately from irritating effect and ultimately from cicatricial effect, and that employment of the negative pole does not prevent this. My study of the subject and the experience it has brought me, digested with all the impartiality I possess, led me to state that the allegation that electricity, however employed, is able to remove organic urethral stricture radically lacks the requirement of demonstration. The confidence of its advocates that it will radically cure organic fibrous stricture is, in my opinion, due to the combined credulity of the patient and imagination of the surgeon, or to some special but fortuitous act of Providence upon the co-operation of which, in the case of his own patients, the general practitioner can not with any confidence rely."

CONCLUSIONS.

1. Dilatation is the best treatment in strictures if it cures.

2. Electrolysis is a modified dilatation; it is a good treatment unless contra-indicated.

3 . Cutting is the cure in non-dilatable cases, and in all strictures at or near the meatus.

100 State Street.

\section{THE RECLASSIFICATION OF SOME ORGANIC NERVOUS DISEASES ON THE BASIS} OF THE NEURON.*

\section{[ABSTRACT.]}

Preseuted to the Section ou Neurology and Nedical Jurisprudence, at the Forty-ninth Annual Meeting of the American Medical Association, held at Denver. Colo., June 7-10, 1898. BY CHARLES K. MILLS, M.D.

A few neurologic writers have already spoken of organic neuronic diseases, and many views and theories, as well as a considerable terminology springing from the doctrine of the neuron have rapidly been developed.

The most important point of the present communi. cation is to direct attention to the fact that the time has arrived for applying the theory of the neuron, or the nerve cell regarded as an anatomic unit in the classification of some of the most important organic nervous diseases, and especially many of those which have been hitherto placed under such heads as system diseases and primary degenerations.

In a general way, the diseases to which I shall chiefly refer may be classified under the three heads of toxe.

5 New York Med. Jour. (1ct. 6, 1888.

*The substance of this paper was read before the William Pepuer Yedical Society of the University of l'ennsylvania. March 11, 1898; and before the Baltimore Neurological Society, A pril 20, 1898; and was oresented to the Section on Neurology and Medical Jurisprudence of the American Medical Association in June, 1898, as above indicated. Just before sending the paper to the last lumed meeting I received a copy of a work by J.M. Gerest, entitled "Les Affections Nerveuses Systimatiques et la Théorie des Neurones," 1898. The author of this work takes in many respects the same points of view as the writer. mias, inflammations and primary degenerations, but more especially the last. In a fundamental sense they might probably all be classed as toxemias, since the chronic scleroses and degenerations, as well as various recognized forms of peripheral and central inflammation are primarily due to a toxic cause, using here the word toxic in its broadest sense.

Accepting the classes of toxemias, inflammations and degenerations, many of these would be better grouped as neuronal or neuronic diseases, rather than as diseases of the brain, spinal cord or of the peripheral nerves, as they have usually been designated.

Not only do toxic agents resulting in inflammations and degenerations tend to attack special systems of neurons; but not infrequently a selective tendency is exhibited as regards the sensory, the motor, the intercalary, or the association systems of neurons; and more than this, special portions of the motor, of the sensory, or of the intermediate chain of neurons are liable to be attacked in particular instances, while other portions of this particular system escape. The facts at our command are chiefly with regard to the sensory and motor projection systems, but the evidence is accumulating that in particular cases commissural, associating and storage systems may also be liable to elected system diseases.

The term "system disease" is one which has long been in use in neurology. Commonly it has been applied to those diseases of the neuraxis, and especially of the spinal cord, which attack, or are supposed to attack, exclusively certain tracts or regions of nerve fibers or nerve cells having common anatomic relations and physiologic properties. It is, however, more scientific and more helpful practically, to consider system diseases from the basis of the neuron, than to regard them as diseases of the cord or of the brain.

One point to be strongly emphasized incidentally is that the toxic agents tend primarily to attack systems of neurons as a whole, and not this or that particular part, as the cell corpus, the axon, the collaterals or terminals. It is true, however, that the destructive influence of the poisoning agent may be first exhibited, so far as can be learned by the means of investigation at our command, in one or in another portion of the nerve cell.

Dr. Spiller and the writer have recently made a study of acute ascending paralysis (Landry's paralysis), in the case of a patient who died in the wards of the Philadelphia Hospital' ${ }^{1}$. A careful postmortem examination was made, this including not only an examination of the organs in general, and of the central nervous system, but also that of some of the motor and sensory nerves. The conclusions reached in this study are of interest in connection with the views here presented. Both the peripheral nerves and the cell bodies were the seat of extensive degeneration.

It has been held by some that in multiple neuritis it is presumable that the poison acts on the entire sensory neuron at the same time, which accords with the views of the writer that in most neuronic diseases of toxic origin the poison tends to attack the neurons elected as a whole.

The disease which has so long been known by such names as locomotor ataxia, tabes, ta bes dorsalis and posterior spinal sclerosis needs, from the standpoint of the neuron, to be presented in a new pathologic light, but I can not review here the recent literature of this sub.

1 Mill, C.K., and Spiller, W.G. : See Jour. of Nerv. and Ment. Dis., vol. xxv, No. 6, June, 1898 . The paper giving the results of this investigation Was read at the meeting of the Am. Neurolog. Assoc., held in New York, May $26,27,28,1898$. 
ject; indeed, this is not the purpose of the present contribution, which is rather to present broad suggestions regarding neuronic classification. Using terms as we now commonly employ them, tabes, like other diseases to which attention will presently be directed, is neither purely spinal nor purely neural, neither purely central nor purely peripheral; but it is an affection primarily of sensory neurons or nerve cells, and Langdon", with others, has very properly suggested to regard it as a "sensory neuron degeneration." "As a result of predisposition, hereditary or acquired, and the action of a toxin or toxins (left by a preceding syphilis, usually though not invariably), the nutrition of the nervous system as a whole is lowered. In certain groups of sensory neurons (muscle sense, iris reflex, optic nerve), the impairment is greater or the resistance of the tissue is less, and in these groups degenerative changes occur."

Tic douloureux and some of the forms of primary degeneration which single out the olfactory, optic, cochlear, vestibular and glossopharyngeal nerves should, in the opinion of the writer, be classified with tabes as sensory neuron degenerations.

Let us now, in the development of the same line of thought, turn our attention to some of the well known forms of "sclerosis" and "degeneration."

Most authors still content themselves with describing the pathology of ataxic paraplegia as sclerosis attacking the lateral columns and part of the posterior columns, the posterior root zones usually escaping disease. The lesions in the so-called spinal form of hereditary ataxia differ from those of acquired ataxic paraplegia in that the lesions are more extensive and involve the posterior root zone in all regions of the cord, and the whole of the posterior columns except the small region to the inner side of the neck of the posterior cornu, the direct cerebellar tract being also involved. It would be better to class these diseases as instances of mixed neuron system degeneration. We have in them degeneration beginning both in the sensory neuron systems and in the motor neuron systems, and in the case of hereditary ataxia involving additional special systems.

In amyotrophic lateral sclerosis the parts early invaded are the terminals and collaterals of the cortical motor neuron, and later the ganglion cells of the ventral horns. Both the direct and crossed pyramidal tracts and some of the systems of short fibers in the lateral columns, as well as the cells of the ventral horns, have been found degenerated in careful postmortem examinations. Somewhat frequently in this disease the nuclei of the cranial nerves and the pyramidal tracts in the oblongata and pons are implicated in the degeneration, and in rarer cases this has been traced upward to the peduncles, and even to the motor cortex. In extremely rare cases even the cells of the motor cortex have been found to take part in the disease. It is not improbable that implication of the entire extent of the cortical motor neuron will be more frequently found when the present methods, advanced as they are, are improved and investigations are more complete. The disease is in reality one attacking two systems of neurons, and at least one of the names used as descriptive of it should indicate this fact.

Those forms of progressive muscular atrophy, either acquired or embryonal which presumably have their origin in the nerve cell, should be classified under the head of motor neuronal degeneration, and then subI angdon, F. W.: Med. Record, N. Y., Jan. S, 189s. classified according to their position and extent. Bulbar paralysis should also be classified under motor neuronal degenerations, and subclassified according to the special cranial nerve system involved. More or less generalized cranial motor neuronal degenerations would include the different types of bulbar paralysis; and motor neuronal degenerations of spocial nerves would give such well known affections as lin. gual atrophy, facial atrophy (here including painless. tic), and the atrophic ophthalmoplegias.

The so-called "primary lateral sclerosis" is something of a stumbling block in our efforts at neuronic classification. According to views long accepted, in this affection the lesion is a degeneration or a sclerosis of both lateral columns, usually beginning in the white matter of the lumbar cord and subsequently passing up the cord, but without involving the cells of the ventral horns. In other words, according to this view we have a primary degeneration attacking and confining itself to the processes of the nerve cells. While this disease has, however, long held its place in the nosology of nervous diseases, only an exceedingly limited number of autopsies apparently uphold the pathology above indicated. The probabilities are altogether against the idea of a true primary lateral sclerosis; and the cases which present the well-known symptomatology of this affection can probably all be otherwise relegated, as to Erb's spinal paralysis, amytrophic lateral sclerosis and hysterical spasmodic paraplegia.

The application of the theory of the neuron to classification is most readily made in the case of the degenerative diseases, and yet the same truths are applicable to diseases which more rapidly run their course, as overwhelming toxemias or active inflammations. In Landry's paralysis the peripheral motor neuron is attacked and largely destroyed. In poliomyelitis, both of the infantile and of the adult type, in the polio-encephalitis superior and inferior of Wernicke, and in the cortical polio-encephalitis of Strümpell, motor neuron systems are attacked. Much controversy has taken place as to whether in multiple neuritis the affection is primarily one of the peripheral nerves (that is, of the axis cylinder processes), of the cell bodies, or of both. Much evidence, clinical and pathological, is certainly in favor of the view that polyneuritis in many cases is a true peripheral disease; but in many other cases those forms of inflammation which are usually designated as neuritis and as poliomyelitis involve the entire neuron, and not simply the peripheral nerve (in the ordinary acceptation of the use of the word "peripheral"), on the one hand, or the cell bodies in the ventral horns on the other. Those forms of so-called polio-encephalitis and polyneuritis which have been most studied are now almost universally conceded to be either of infectious or toxic origin. With regard to multiple nouritis it is no new thing for me to teach the involvement of both nerve and cord in a large number, if not in a majority, of the cases. Long before the theory of the neuron was thought of, I expressed my belief that in many cases. of so-called multiple neuritis both periphery and spinal centers were implicated, in other words, as then expressed, we had a concurrence of multiple neuritis and of generalized poliomyelitis in the same case. Several recent investigators have shown involvement of the posterior roots and even of the entire sensory and motor neurons both in multiple neuritis and in cerebrospinal meningitis. Long ago I also 
recorded clinical observations on cases of multiple neuritis which eventually became cases of true tabes. Cases of poliomyelitis with implication of the peripheral motor nerves are also on record.

These diseases are, therefore, in some cases at least, neither instances of neuritis in a strict sense or of poliomyelitis. They are perhaps what might be termed "nouronitis," and this term has been suggested, but seems to have an unnatural sound even to a neurological ear.

While the facts which point to the importance of reclassifying certain diseases on the basis of the neuron are clear and convincing, it is difficult to determine on a simple and practical method of doing this. It will probably be best for clinical reasons to arrange these diseases in the first place under the heads of 'mbryonal and of acquired neuronal diseases, and then to subclassify these according to the particular system or systems of neurons attacked. This subdivision would include (1), diseases of the sensory neuron systems; (2), diseases of the motor neuron systems; (3), diseases of both sensory and motor neuron systems at the same time; (4), diseases of intercalary or associating systems of neurons; and (5), diseases in which numerons systems of neurons are more or less indiscriminately attacked. The fourth and fifth categories will receive only passing attention in this paper. Within these groups an effort could of course be made to subclassify toxemias, inflammations and degenerations, but this will not be attempted in the present paper, and perhaps it would not answer any useful purpose to make the attempt. By far the larger number of neuronal affections are perhaps to be classed under the general head of primary degenerations, at least with our present pathological knowledge; but toxemias and inflammations which do not lead to degeneration are also, as I have already indicated, to be included.

Every thinking neurologist has felt the importance of having some good method of classifying under some general head the numerous hereditary or family affections. The theory of the neuron offers us one of the best methods of doing this. The embryonal or family diseases can be classified with some approach to success under the different physiologic systems of neurons. Under primary embryonal degenerations of motor neuron systems, for instance, such affections as Little's disease, and perhàps other forms of infantile diplegia or hemiplegia, and some of the forms of juvenile muscular atrophy might be grouped; under embryonal degenerations of sensory or of mixed sensory and motor neuron systems might be included Friedreich's ataxia, hereditary cerebellar ataxia, and the common type of syringomyelia; and under special forms of embryonal neuronal degenerations could be classed amaurotic family idiocy, so-called Huntingdon's chorea, for which Halleck has suggested the name of dementia choreica, and even perhaps some of the special types of the insanity of developmental periods.

Amaurotic family idiocy is a type of affections in which numerous systems of neurons are arrested in their development, while general paralysis of the insane is a type of acquired disease in which numerous systems of neurons are more or less indiscriminately attacked. Hirt, by classifying this disease under the head of general diseases of the nervous system, recognized the importance of taking it from the category of cerebral affections or of ordinary insanities.
Provisionally the views expressed in the previous pages might be in part summarized in tabular form as follows: EMBRYONAL NEURONAL DEGENERATIONS.

I. Sensory or sensorimotor types. 1, Friedreich's ataxia; 2 , hereditary cerebellar ataxia; 3 , syringomyelia.

II. Motor types. 1, spastic diplegia (some types, as Little's disease) ; 2, progressive muscular atrophy (some types).

III. Mixed and multiple types. 1, amaurotic family idiocy; 2 , dementia choreica (Huntingdon's chorea); 3 , developmental insanity (some types).

ACQUIRED NEURONAL DEGENERATIONS.

I. Sensory types. 1 , tabes; 2 , tic douloureux; 3 , primary degeneration limited to nerves of the special senses.

II. Motor types. 1, progressive muscular atrophy (some types); 2, amyotrophic lateral sclerosis; 3, progressive bulbar paralysis ; 4, primary degeneration of particular motor nerves, as of the hypoglossal, facial, trigeminal and ocular.

For the present it will perhaps be best to place neuronal inflammations and toxemias in a special class, including, 1, acute ascending paralysis (Landry's paralysis); 2, anterior poliomyelitis ; 3 , polio-encephalitis superior and inferior of Wernicke; 4, cortical polio-encephalitis of Strümpell ; 5 , multiple neuritis and neuromyelitis.

\section{HOW DOES THE CAUSE OF DISEASE PRO- DUCE DISEASE? A NEW INTER- PRETATION OF OPERATIVE PRINCIPLES.}

\section{BY W. R. DUNHAM, M.D.} KEENE, N. H.

The following direction of research was suggested by J. M. DaCosta, M.D., LL.D., in his address before the Association of American Physicians, at Washington, D. C., May 4, 1897: "The great advance of our time it seems trite to say, is the ardent study of the causation of disease. And often what we consider the cause, may thus be only the consequences of disease. The soil and the forces, too, are to be studied and critically examined."

There has been much written respecting the soil of disease, while it is a new suggestion that we critically study the nature of the forces which present disease phenomena. And with such review it may be well to mention, that the science-mind for many generations has been satisfied with the interpretation; that forces from without, that active medical principles and active causes of disease in addition to active vital forces, have taken part in the operations presented by the living human organism, while of recent date the more advanced thinkers deny that supplementary forces can be introduced to operate the machinery of life. Such expectancy, however, has prevailed for a long period and will doubtless be slow of elimination until a clear comprehension is developed respecting the nature of the vital forces.

A distinguished editor of an American medical journal states, as late as January, 1898: "There is no drug yet discovered, unless it be alcohol, which adds to the forces of the body." But even this one source will be discredited when it is recognized that medicobiologic science is based entirely on the functions and operative principles of the vital force energies. Thus in the near future a different operative plan of medical science will be taught in the schools, effecting as great a revolution of ideal molus operandi as was experienced in the acceptance of the Copernican interpretation of astronomic plan. Therefore, in place of teaching medico-biologic science as being based on functions and operatıve principles, derived from both within and without, this division of natural science will have its recognized first principles begin- 